\title{
High-sensitivity detection of cryptic Wolbachia in the African tsetse fly (Glossina spp.)
}

\author{
Daniela I Schneider ${ }^{1,2}$, Andrew G Parker $^{3}$, Adly M Abd-alla ${ }^{3}$ and Wolfgang J Miller ${ }^{1 *}$
}

\begin{abstract}
Background: In African tsetse flies Glossina, spp. detection of bacterial symbionts such as Wolbachia is challenging since their prevalence and distribution are patchy, and natural symbiont titers can range at levels far below detection limit of standard molecular techniques. Reliable estimation of symbiont infection frequency, especially with regard to interrelations between symbionts and their potential impact on host biology, is of pivotal interest in the context of future applications for the control and eradication of Glossina-vectored African trypanosomosis. The presence or absence of symbionts is routinely screened with endpoint polymerase chain reaction (PCR), which has numerous advantages, but reaches its limits, when detecting infections at natural low titer. To not only determine presence of native tsetse symbionts but also to localize them to specific host tissues, fluorescence in situ hybridization (FISH) can be applied. However, classic FISH assays may not detect low-titer infections due to limitations in sensitivity.

Results: We have compared classic endpoint PCR with high-sensitivity blot-PCR. We demonstrate that the latter technique allows for clear detection of low-titer Wolbachia in the morsitans and palpalis groups while classic endpoint PCR does not. In order to localize Wolbachia in situ in high and low-titer Glossina species, we applied high-end Stellaris ${ }^{\circledR}$ rRNA-FISH. We show that with this high sensitivity method, even low amounts of Wolbachia can be traced in specific tissues. Furthermore, we highlight that more tissues and organs than previously recorded are infested with Wolbachia in subspecies of the morsitans and palpalis groups.

Conclusions: Our results demonstrate that overall symbiont infection frequencies as well as the presence in specific host tissues may be underestimated when using low-sensitivity methods. To better understand the complex interrelation of tsetse flies and their native symbionts plus the pathogenic trypanosomes, it is important to consider application of a broader range of high-sensitivity detection tools.
\end{abstract}

Keywords: Wolbachia, Low-titer symbiont detection limit, Stellaris ${ }^{\oplus}$ fluorescence in situ hybridization, Tissue tropism

\section{Background}

In the African tsetse fly (Glossina spp., Diptera: Glossinidae), detection of bacterial symbionts such as Wolbachia is challenging since their prevalence and distribution are patchy [1], and their natural titers can range at levels far below detection limit of standard molecular techniques $[2,3]$. Reliable estimation of symbiont infection frequency, however, especially with regard to interrelations between the symbionts and their potential impact on host biology, is of pivotal interest in order to control and

\footnotetext{
* Correspondence: wolfgang.miller@meduniwien.ac.at

'Department Cell and Developmental Biology, Center for Anatomy and Cell

Biology, Medical University of Vienna, Vienna, Austria

Full list of author information is available at the end of the article
}

eradicate African trypanosomosis in the future [4]. While classic endpoint PCR has numerous advantages, it soon reaches its limits, when it comes to detecting very low loads of symbionts (low-titer infections), and localize the symbionts to specific host tissues (tissue tropism). Classic fluorescence in situ hybridization (FISH) using $16 S$ rRNA oligo probes is the method of choice for following tissue tropism. However, sensitivity may be too low for symbiont detection in biologically relevant host organs like testes but also for the in situ detection of generally low-titer infections such as the ones of G. $p$. gambiensis. In the presented study, we have compared the feasibility of classic PCR and blot-PCR for low-titer Wolbachia in species and subspecies of the genus 
Glossina. Furthermore, we took advantage of the novel Stellaris ${ }^{\circ}$ technology to trace such low-titer Wolbachia in situ. As known from recent studies, not only hightiter infections, but also Wolbachia low-titer infections impact host biology [5]. Hence, their reliable detection with particular regard to their location in situ, is important for better understanding host-symbiont relations plus the crosstalk with trypanosome parasites.

\section{Results}

\section{Low-titer Wolbachia infections in Glossina spp. may be overlooked with standard endpoint-PCR techniques}

To demonstrate the advantage of more sensitive detection methods over standard techniques, we employed and compared two endpoint PCR techniques plus one high-end blot-PCR. First, we tested the classic Wolbachia marker wsp (Wolbachia outer surface protein gene) with Glossina subspecies from the palpalis and morsitans groups (Table 1).

Endpoint PCR using the single copy wsp marker clearly detects high-titer Wolbachia in females and males of G. m. morsitans (Gmm) and G. m. centralis $(G m c)$, but G. swynnertoni (Gsw) and G. p. gambiensis $(G p g)$ seem uninfected. We did also not detect Wolbachia in the two subspecies G. p. palpalis (Gpp) and G. f. fuscipes (Gfu), which were previously reported Wolbachia-negative (Fig. 1a) [1]. Next, we applied the multicopy locus ARM (Wolbachia A-supergroup repeat motif) [3] to the same sample set (Fig. 1b). Similar to

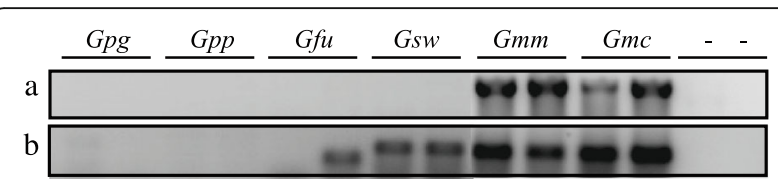

Fig. 1 Detection of Wolbachia in Glossina females and males via polymerase chain reaction (PCR). a Wolbachia-specific single copy wsp-PCR detects the symbiont only in high-titer $\mathrm{Gmm}(q, \delta)$ and Gmc $\left(+, \delta^{\lambda}\right)$. b The more sensitive multicopy ARM-PCR additionally detects WGsw (Wolbachia of G. swynnertoni). In the Wolbachiauninfected G. f. fuscipes, ARM-PCR amplifies a possibly nonspecific product of smaller size. Negative controls are Wolbachia-uninfected Drosophila simulans, NouméaTC (Dsim ${ }^{\text {minus }}$ ) and non-template control (NTC). Females are first on gel. Each PCR was at least repeated once in order to confirm results. Abbreviations: wsp Wolbachia outer surface protein gene

wsp-PCR, the more sensitive multicopy specific ARMPCR easily detects high-titer Wolbachia of Gmm and Gmc. In addition, it traces the earlier described low-titer infection in Gsw [3]. Interestingly ARM-PCR successfully amplifies a smaller fragment in Gfu males but not females, (Fig. 1b; and see below).

\section{High-end blot-PCR increases Wolbachia detection limit}

As demonstrated above, wsp and ARM markers are efficient tools to screen for Wolbachia infection status when symbiont titers are at high or medium levels. In order to further increase the detection limit of our assay, we employed a combined PCR-hybridization (blot-PCR) assay $[2,6]$. This method has proven reliable in detecting

Table 1 List of Glossina and Drosophila strains analyzed in this study

\begin{tabular}{|c|c|c|c|c|c|c|c|c|}
\hline & Species & $\begin{array}{l}\text { Species } \\
\text { group }\end{array}$ & $\begin{array}{l}\text { Strain/ } \\
\text { abbr. }\end{array}$ & Origin/reference & & & & \\
\hline \multirow[t]{4}{*}{$\begin{array}{l}\text { Drosophila } \\
\text { strains }\end{array}$} & Drosophila simulans & simulans & NouméaTC & [18] & \multicolumn{4}{|c|}{$\begin{array}{l}\text { Wolbachia negative } \\
\text { control }\end{array}$} \\
\hline & \multirow[t]{3}{*}{ Drosophila willistoni } & \multirow[t]{3}{*}{ willistoni } & \multirow[t]{3}{*}{ P98 } & \multirow[t]{3}{*}[19]{} & \multirow{2}{*}{\multicolumn{4}{|c|}{$\begin{array}{l}\text { Wolbachia positive } \\
\text { control } \\
\text { Wolbachia status } \\
\text { (experimental) }\end{array}$}} \\
\hline & & & & & & & & \\
\hline & & & & & wsp & $A R M$ & blot & $\mathrm{FISH}$ \\
\hline \multirow[t]{6}{*}{ Glossina strains } & Glossina morsitans morsitans & morsitans & $\mathrm{Gmm}$ & $\begin{array}{l}\text { Takáč Lab, Slovak Academy of Sciences, Bratislava, } \\
\text { Slovakia }\end{array}$ & $\begin{array}{l}++ \\
+\end{array}$ & $\begin{array}{l}++ \\
+\end{array}$ & $\begin{array}{l}++ \\
+\end{array}$ & $\begin{array}{l}++ \\
+\end{array}$ \\
\hline & Glossina morsitans centralis & morsitans & Gmc & $\begin{array}{l}\text { Insect Pest Control Laboratory FAO/IAEA, Vienna, } \\
\text { Austria }\end{array}$ & $\begin{array}{l}++ \\
+\end{array}$ & $\begin{array}{l}++ \\
+\end{array}$ & $\begin{array}{l}++ \\
+\end{array}$ & $\begin{array}{l}++ \\
+\end{array}$ \\
\hline & Glossina swynnertoni & morsitans & Gsw & $\begin{array}{l}\text { Insect Pest Control Laboratory FAO/IAEA, Vienna, } \\
\text { Austria }\end{array}$ & - & ++ & ++ & nd \\
\hline & Glossina palpalis palpalis & palpalis & $G p p$ & $\begin{array}{l}\text { Insect Pest Control Laboratory FAO/IAEA, Vienna, } \\
\text { Austria }\end{array}$ & - & - & - & - \\
\hline & $\begin{array}{l}\text { Glossina palpalis } \\
\text { gambiensis }\end{array}$ & palpalis & Gpg & $\begin{array}{l}\text { Insect Pest Control Laboratory FAO/IAEA, Vienna, } \\
\text { Austria }\end{array}$ & - & - & + & + \\
\hline & Glossina fuscipes fuscipes & palpalis & Gfu & $\begin{array}{l}\text { Insect Pest Control Laboratory FAO/IAEA, Vienna, } \\
\text { Austria }\end{array}$ & - & - & $+?$ & nd \\
\hline
\end{tabular}

Two Drosophila strains were used as Wolbachia-positive and -negative controls (P98, NouméaTC). The table lists all Glossina strains used for experiments, including Wolbachia infection status based on PCR (Wolbachia outer surface protein gene, Wolbachia A-supergroup repeat motif,); blot-PCR using a wsp probe (listed as 'blot'), and fluorescence in situ hybridization with Wolbachia 16-23S rRNA probe. Wolbachia infection titer is indicated by ' + ' (low), ' ++ ' (intermediate), and ' +++ ' (high). Abbreviations: ws $p$ Wolbachia outer surface protein gene, ARM Wolbachia A-supergroup repeat motif, FISH fluorescence in situ hybridization, $n d$ not determined 
low loads of Wolbachia, which were undetected in standard PCR assays such as the wsp-based PCR [2, 5, 6]. As shown in Fig. 2b consequent hybridization with an internal Wolbachia-specific wsp-probe allows more Wolbachia to be traced than in the wsp-PCR assay shown in Fig. 2a. Similar to classic endpoint wsp-PCR, blot-PCR detects high-titer Wolbachia of Gmm and Gmc (even before hybridization; shown in Fig. 2a). It further allows detection of the symbiont in Gpg females (Fig. 2b, black arrow), and both sexes of Gsw (Fig. 2b, black arrows). Also, Gfu, which was reported Wolbachia-uninfected previously [1], seems to amplify a weak $w s p$-band on the blot (Fig. 2b, black arrow), although it is very faint and its reliability should thus be questioned.

\section{High-end Stellaris ${ }^{\oplus}$ rRNA-FISH helps to detect low-titer Wolbachia infections in situ}

In order to localize Wolbachia in situ, we tested various Glossina species via fluorescence in situ hybridization (FISH). As standard FISH is not sensitive enough to detect low-titer Wolbachia (Schneider, unpublished) we employed high-end Stellaris $r$ RNA-FISH. This method uses a set of up to 48 oligonucleotides, which collectively bind along the same target transcript (see Methods section for probe design). First, we tested in situ localization of high-titer Wolbachia in Gmm and Gmc (Fig. 3). As shown in Fig. 3, the symbionts are present in the reproductive organs of females and males (Fig. 3a, b) plus in the sheath cells of the spermatheca (Fig. 3c, f) in both high titer hosts. Wolbachia also infect adipocytes, the main fat body cells (Fig. 3d), and the milk glands (Fig. 3e). The latter tissue also houses tsetse's primary symbiont Wigglesworhtia, as shown via costaining with symbiont-specific $16 S$ probe (Fig. 3e').

So far, the presence of Wolbachia in male gonads of Glossina was never reported in the literature [7]



although Wolbachia from Gmm and Gmc are well known for causing strong cytoplasmic incompatibility (CI) $[2,8]$. By using high-end Stellaris ${ }^{\circ} r$ RNA-FISH, however, we clearly detected the symbiont in the testes of both Gmm and Gmc. Most interestingly, we did not observe overall infection of the reproductive organ, but a strong restriction to certain stages of spermatogenesis (Fig. 4). We detected Wolbachia in the hub of the testis in $\mathrm{Gmm}$, which is formed by non-dividing stromal cells connected to the surrounding stem cells (Fig. 4a). Further, Wolbachia are present in a later stage of spermatogenesis, the so-called 64 'onion-stage' spermatids (Fig. 4b).

We further analyzed in situ localization of Wolbachia in the low-titer species G. p. gambiensis (Gpg), and could clearly detect the symbiont in ovaries (Fig. 5a). Similarly, to the situation in the high-titer species $G m m$ and $G m c$, Wolbachia was localized to the sheath cells of the spermatheca (Fig. 5c). Besides that, Wolbachia are also present in the spermathecal duct (Fig. 5b, c). So far, we were not able to elucidate the presence of Wolbachia in males of low-titer Glossina species unambiguously.

\section{Discussion}

Detection of Wolbachia inside the host can be challenging when symbiont loads are low. In such cases, detection techniques of higher sensitivity are required to avoid false negative typing of specimens. We have compared in different species and subspecies of Glossina, the feasibility of single copy Wolbachia-specific PCR (wsp) with an improved multicopy locus assay $(A R M)$. The standard Wolbachia outer surface protein gene (wsp) PCR is commonly used to detect and type Wolbachia in various host species, but has the disadvantage of low detection limit when it comes to low-titer infections. For the tsetse fly samples where standard wsp-PCR was not sufficient to detect Wolbachia, the more sensitive $A R M$-marker, however, enhanced the detection sensitivity and thus detected low-titer Wolbachia infection in Gsw. This finding is a confirmation of previous results on the feasibility of ARM for low-titer Wolbachia infections [3]. However, Wolbachia of Gpg, Gpp and Gpal could not be traced with $A R M$ in laboratory-reared flies, although the presence of Wolbachia in these species was reported previously in field collected samples [1]. An explanation for this observation could be the complete lack of the ARM motif from the genomes of these Wolbachia strains. Gpg falls into a different species group than Gpal, Gmm and Gmc, which could account for differences in the associated Wolbachia strains, such as presence or absence of a repeat motif. Alternatively, the Wolbachia titer could simply be below the detection limit of $A R M-\mathrm{PCR}$ in these species. Finally, we could not detect any Wolbachia in Gfu and Gpp using ARM-PCR, 

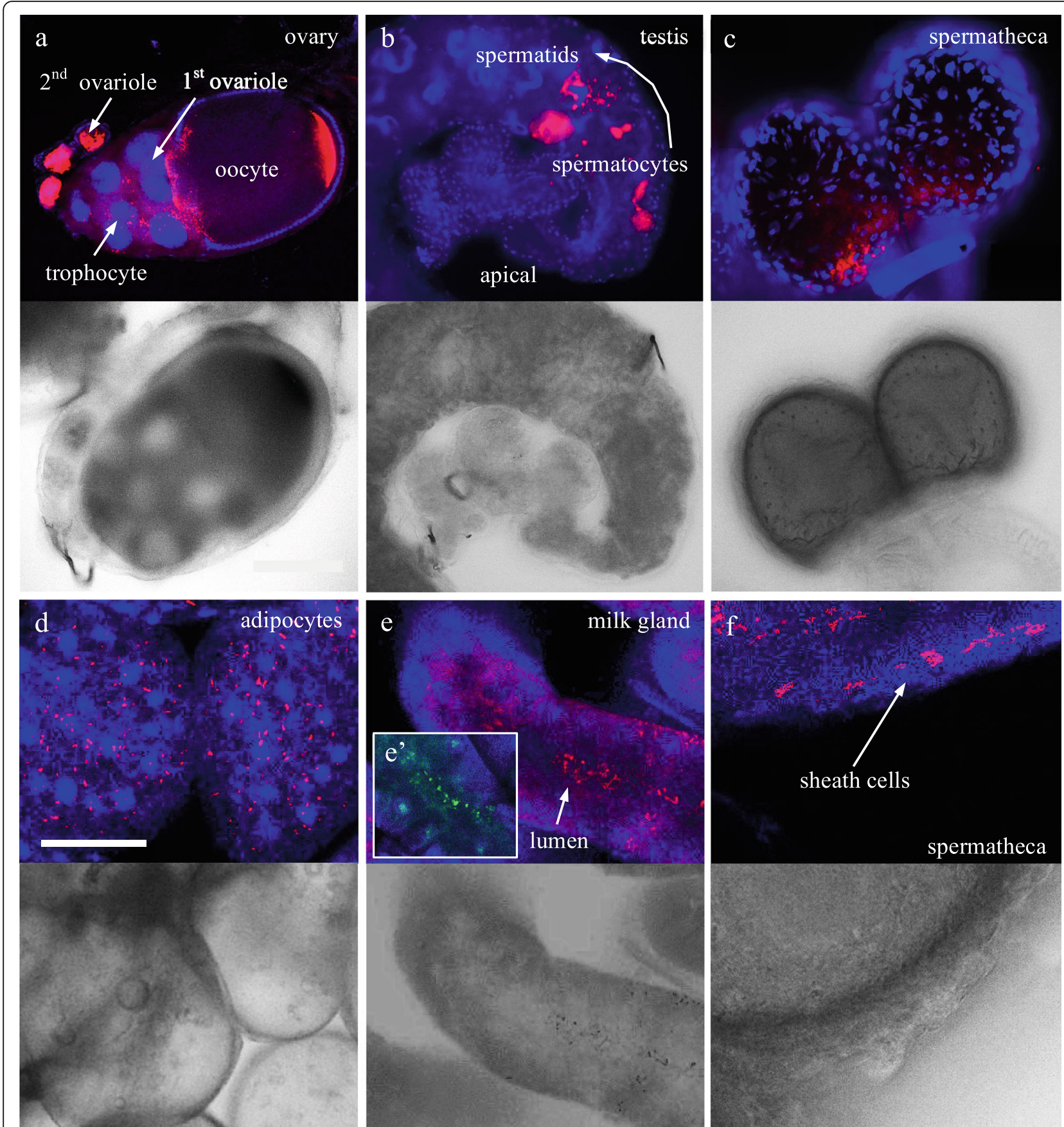

Fig. 3 Stellaris ${ }^{\oplus}$ rRNA-FISH on high-titer Glossina species (G. m. morsitans, G. m. centralis). a In the ovary Wolbachia are present in the first and in the second ovariole plus at the posterior pole of the oocyte. $\mathbf{b}$ Wolbachia are not detected in early stages of spermatogenesis (hub cells, spermatogonia) but are visible in later stages (spermatocytes, spermatids). Also the spermatheca, particularly the sheath cells $(\mathbf{c}, \mathbf{f})$, as well as adipocytes (d) and milk gland (e) are infected with Wolbachia. (e') Wigglesworthia, primary symbiont of Glossina, are also present in the milk gland. Wolbachia are stained in pink (16-23S rRNA), Wigglesworthia are shown in green (16-23S rRNA), Glossina DNA is stained in blue (4',6diamidino-2-phenylindole), brightfield images for better orientation are presented for a-f. Scale bar is $100 \mu \mathrm{m}$

which agrees with a previous study that reported those species uninfected ([1] and references therein). In Gfu males, however, we found a smaller ARMfragment, which could be explained by a potential degenerated Y-chromosomal copy of the motif. This assumption though needs to be tested by further sequencing experiments.

We recently reported on the advantage of the multicopy $A R M$-marker over blot-PCR, due to less time consumption (no blotting, hybridization and detection 


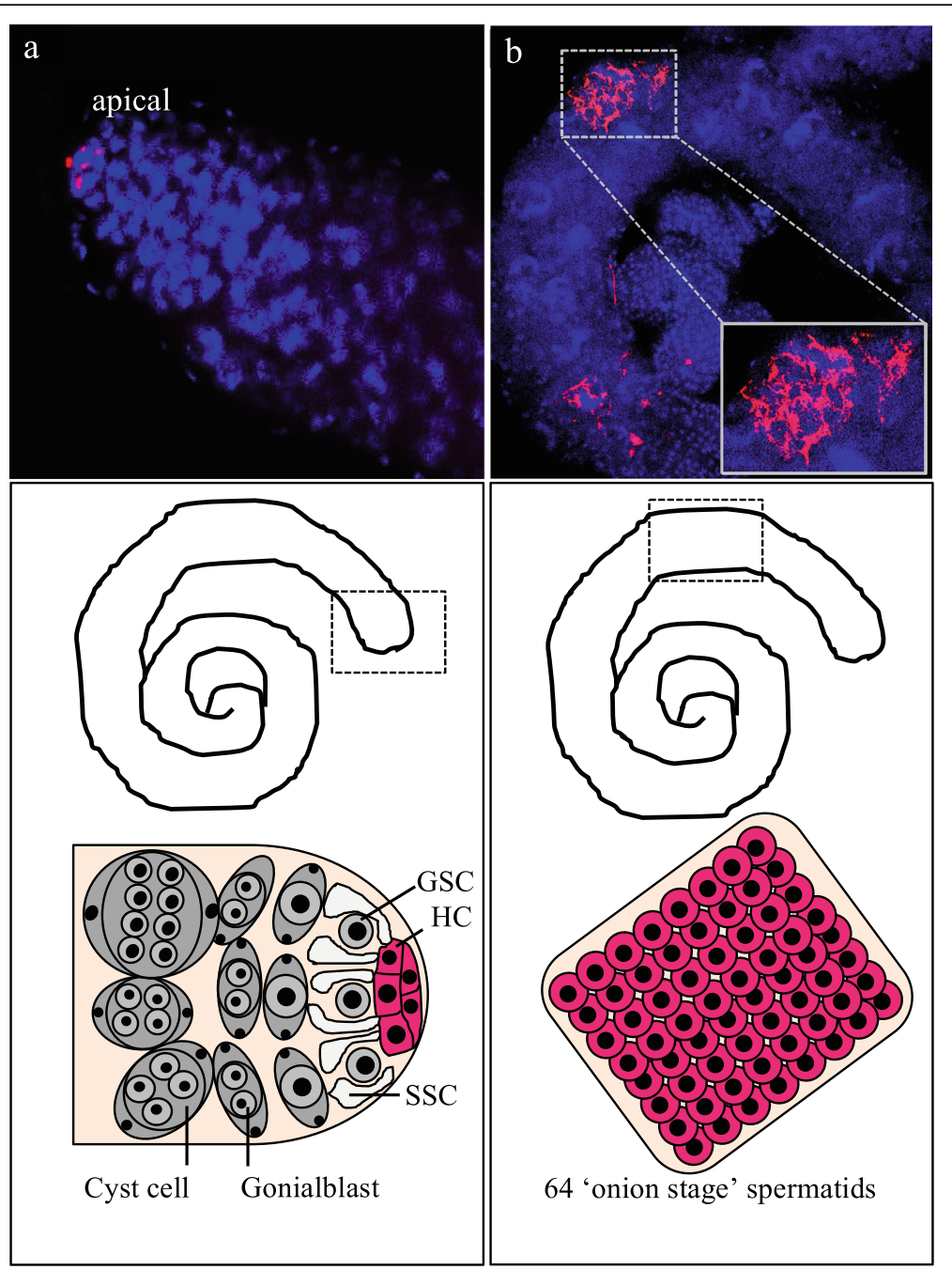

Fig. 4 Stellaris $^{\oplus}$ rRNA-FISH on testes of high-titer G. m. morsitans. a Wolbachia accumulating in the hub cells of the apical part (indicated by dotted box) of the testis. $\mathbf{b}$ In later stages of spermatogenesis, Wolbachia are present in 64 'onion stage' spermatids. Wolbachia are stained in pink (16-23S rRNA), Glossina DNA is stained in blue (4',6-diamidino-2-phenylindole). Minimum three individuals from each sex were dissected and processed for FISH. Abbreviations: GSC germline stem cells, SSC somatic stem cells, HC hub cells
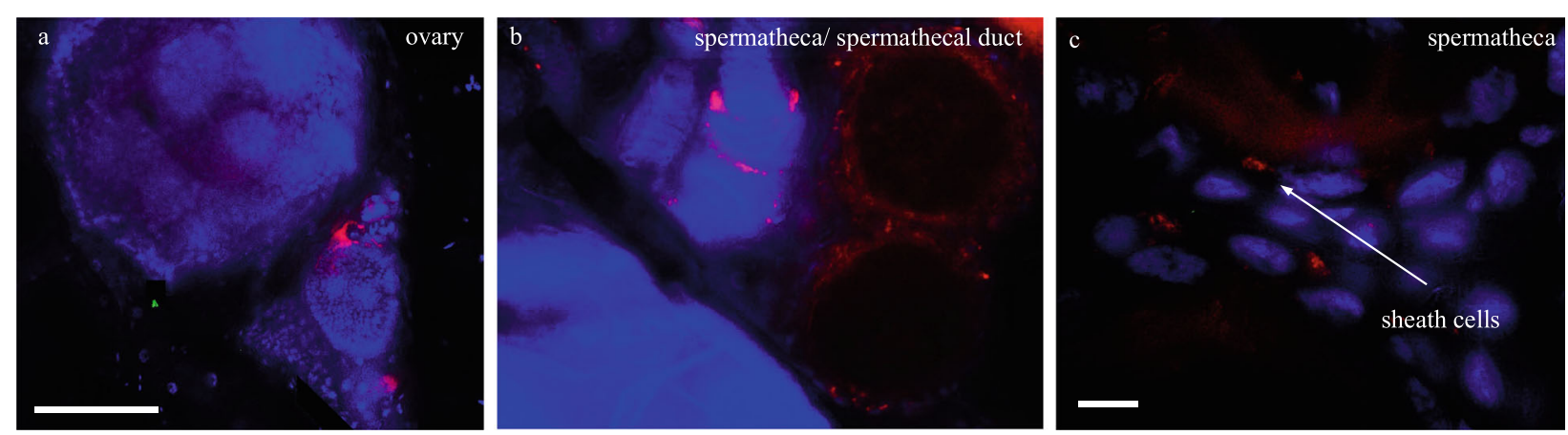

Fig. 5 Stellaris ${ }^{\oplus}$ rRNA-FISH on low-titer G. p. gambiensis (Gpg). Wolbachia are present in (a) ovaries, b spermatheca, spermathecal duct, and (c) in the sheath cells of the spermatheca. Wolbachia are stained in pink (16-23S rRNA), Glossina DNA is stained in blue (4,,6-diamidino-2-phenylindole). Scale bars are $100 \mu \mathrm{m}(\mathbf{a}, \mathbf{b})$ and $10 \mu \mathrm{m}(\mathbf{c})$ 
steps) but higher sensitivity, at least for Drosophila specimens [2]. Furthermore, the ARM multicopy target variant seems to be restricted to A-supergroup Wolbachia strains and absent in the others [3]. However, while the previous study focused on Drosophila, we report on various Glossina subspecies in the present study, and for the tested Glossina specimens, ARM does not have an advantage over blot-PCR. In contrast, the blot technique confirms an earlier study, which claims an increased sensitivity for Wolbachia by up to 1000-fold [6]. As mentioned before, the reason for this might be very low copy number or absence of the ARM motif from Wolbachia in certain subgroups within the genus Glossina.

Most unexpectedly, we detected a weak Wolbachia signal in Gfu females via blot-PCR. This is interesting as this subgroup was reported uninfected previously [1]. However, it is possible that Wolbachia is indeed present in Gfu, but in 'ultra-low' amounts and is hence very difficult to detect or as a translocated chromosomal copy. The latter event is not unlikely since the presence of multiple extensive chromosomal insertions of Wolbachia into the host genome (chrWol) was reported in Gmm [1, 9, 10]. Furthermore, there may be a variation in Wolbachia infection frequencies between populations of Gfu. Hence, larger numbers of individuals, preferentially from lab colonies as well as from different field collections, need to be tested in order to confirm the presence or absence of low-titer Wolbachia.

It was highlighted recently that not only the titer of symbionts per se, but especially the localization of them inside the host, is important in the context of interactions between the two [2,11-13]. Hence, detecting the symbiont's location in specific tissues and organs of the host is an important aspect of host-symbiont studies. To address this question in Glossina subspecies, we employed high-end Stellaris ${ }^{\bullet}$ RNA-FISH. As anticipated we detected massive Wolbachia infestation in gonads of high-titer Gmm and $G m c$ females. However, we also found Wolbachia in lumen and secretory cells of the milk gland of $G m m$, along with the primary tsetse fly symbiont Wigglesworthia (Fig. 3e'). This is in contrast to an earlier report, which showed that the milk gland is infected by the two other tsetse symbionts, Wigglesworthia and Sodalis, but not by Wolbachia [7]. The presence of Wolbachia in the milk gland may point towards a yet undiscovered alternative transmission route from mother to offspring, similar to Wigglesworthia transmission (reviewed in $[14,15])$. Since the developing larva feeds on milk lactated from the milk gland during its intrauterine development, it is tempting to speculate that the Wolbachia of this viviparous host system might not only be transmitted trans-ovarially but also nutritionally to the offspring.

Another tissue that was previously reported Wolbachia-uninfected is the fat body, mainly composed of adipocyte cells [7]. In contrast to that earlier finding, we clearly detected Wolbachia in these cells by using Stellaris $r$ RNA-FISH. Adipocytes play an important role in insect metabolism as they store and release energy in response to the energy demands of the insect [16]. Hence, it is possible that Wolbachia found a way to exploit this host-provided energy source for their own purpose. However, this and their milk gland tropism have to be further elucidated in more detailed studies.

As Wolbachia are transmitted maternally, the presence of the symbionts in male tsetse flies has not been tested and/or reported so far. However, a recent study demonstrated that Sodalis, the second maternally transmitted facultative symbiont of Glossina, are transmitted paternally too [17]. In the context of this study, the detection of high-titer Wolbachia in Gmm and Gmc males via FISH is particularly interesting. It pinpoints that Wolbachia not only persist in male tsetse flies, but are obviously restricted to certain stages of spermatogenesis in the testis. The importance of such host-derived restriction of the symbiont was recently reported in other systems and tissues [11-13]. Moreover, the detection of Wolbachia in the male reproductive organ is important in relation to the capacity of Wolbachia strains to CI. This is in accordance with recent reports on the ability of Wolbachia from Gmc and Gmm to trigger CI [2, 8].

Classic FISH is not sufficient to detect low-titer Wolbachia in Glossina subspecies (data not shown). However, with the help of Stellaris ${ }^{\circ}$ RNA-FISH technique, we could show that Wolbachia are present in the reproductive tissues of Gpg. This finding confirms our data from blot-PCR, where we could also detect Wolbachia in Gpg. The presence of Wolbachia in the uterus has not been reported previously [10] and it remains to be elucidated why the symbionts are accumulating in this tissue.

\section{Conclusion}

Here we demonstrate that detection of symbionts inside the host requires various tools. Low-titer infections can only be detected with high-sensitivity methods such as combined PCR and hybridization. Furthermore, we show that high-end Stellaris ${ }^{\circ}$ technology helps to uncover the localization of Wolbachia to tissues that were previously characterized as uninfected. We also highlight how feasible this method is to trace low-titer Wolbachia in two Glossina subspecies.

Taken together these results demonstrate that choosing a sensitive detection tool for determining symbiont infection status is very important. Gaining deeper insight into the biological and functional interactions between Glossina species, their native symbionts and the pathogenic trypanosomes, we should not underestimate their actual infection frequencies and infection localization through applying only low sensitivity detection tools. 


\section{Methods}

\section{Fly strains}

In this study, we employed Glossina species from the palpalis group (G. palpalis palpalis - Gpp, G. palpalis gambiensis - Gpg, G. f. fuscipes - Gfu), the morsitans group (morsitans morsitans - Gmm, G. morsitans centralis - Gmc, G. pallidipes - Gpa, G. swynnertoni - Gsw), and from the fusca group (G. brevipalpis - Gbr). The Wolbachia-uninfected strain Drosophila simulans, NouméaTC [18] served as negative control for experiments. The Wolbachia-positive control was D. willistoni, Panama98 (P98), carrying native high-titer Wolbachia ( $w$ Wil; [19]). Strains used in this study are summarized in Table 1.

\section{Detection and quantification of Wolbachia}

DNA was extracted from pools of 5-15 Drosophila or a single adult tsetse fly. Individuals were disrupted with a TissueLyser TL bead mill, and DNA was consequently extracted using Puregene chemistry (Qiagen, Germany). Extracts were stored at $-20{ }^{\circ} \mathrm{C}$ until used for experiments. For classic endpoint-PCR, the Wolbachia-specific outer surface protein gene $(w s p)$ was used to determine infection status. Diagnostic wsp-PCR reactions were performed as described in [20]. In addition to the single-copy wsp marker, the multi copy $A R M$ locus (Wolbachia A-supergroup Repeat Motif) was used to screen for Wolbachia infection [3]. Endpoint-PCR combined with hybridization (blot-PCR) was performed as described in [2, 6]. Briefly, Wolbachia-specific $w s p$-PCR was run on genomic DNA samples from all three species groups followed by gel electrophoresis on a $1 \%$ agarose gel. Amplicons were then transferred on a positively charged nylon membrane via capillary blotting, cross-linked and finally hybridized overnight with a digoxygenin-labeled internal $w s p$-probe. Detection was performed using sheep anti-DIG-conjugate (Roche, Germany).

\section{High-end Stellaris ${ }^{\circledR}$ rRNA-fluorescence in situ hybridization (FISH)}

Custom probe sets against Wolbachia $16 S-23 S$ ribosomal RNA transcripts were designed using the Stellaris ${ }^{\oplus}$ FISH probe designer (www.biosearchtech.com/support/tools/ design-software/stellaris-probe-designer; Additional file 1: Table S1). Additional probes targeting 16S-23S rRNA transcripts of Wigglesworthia and Sodalis were designed. We have employed triple staining and FISH on Wolbachia-uninfected plus tetracycline-treated flies to test for cross-reaction of probes with each other and with other bacteria (Additional file 2: Figure S1). Tissues for FISH (ovary, spermatheca, uterus, milk gland, testis, gut) were dissected in sterile $1 \times$ phosphate-buffered saline (PBS) under a dissection microscope, stored on ice until enough material was collected and finally washed with ice-cold $1 \times$ PBS. Tissues were fixed $(3.7 \%$ formaldehyde, $1 \times$ PBS,
$0.15 \%$ Triton-X 100; nuclease-free water) for $20 \mathrm{~min}$ at room temperature followed by three washes for $10 \mathrm{~min}$ each in $1 \times$ PBS with $0.03 \%$ Triton-X 100 (PBS-T). PBS-T was replaced by absolute ethanol and samples were permeabilized overnight under constant agitation at $4{ }^{\circ} \mathrm{C}$. Tissues were washed for $5 \mathrm{~min}$ with washing buffer $(2 \times$ saline sodium citrate [SSC], $10 \%$ deionized formamide, nuclease-free water) at room temperature and then hybridized overnight at $37{ }^{\circ} \mathrm{C}$ in $50 \mu$ hybridization mix (0.1\% dextrane sulfate, $2 \times$ SSC, $10 \%$ deionized formamide, nuclease-free water and $2.5-3$ pmol of each Stellaris ${ }^{\circ}$ DNA-probe (Biosearch Technologies, USA). Wolbachia probe is described in detail in Additional file 1: Table S1. Post-hybridization, tissues were quickly rinsed in washing buffer at room temp $(2 \times 5 \mathrm{~min})$, followed by two washes for $30 \mathrm{~min}$ each at $37{ }^{\circ} \mathrm{C}$. After washing, tissues were equilibrated in $1 \times$ PBS with $0.01 \%$ Triton-X 100 for 5 min at room temperature and then incubated with Alexa Fluor 488 phalloidin (Invitrogen, USA) recognizing F-actin (1:100 in $1 \times$ PBS-T) for $1.5 \mathrm{~h}$. After one quick washing step with $1 \times$ PBS-T, tissues were stained with 4',6-diamidino-2-phenylindole $(0.2 \mu \mathrm{g} / \mathrm{ml}$ in $2 \times \mathrm{SSC})$ for $10 \mathrm{~min}$ at room temperature, washed $2 \times$ with $1 \times \mathrm{SSC}$ and finally mounted in Roti-Mount@ (Carl Roth, Germany) on sterile slides. Visualization of hybridized tissues was performed on either a Nikon A1 or an Olympus Fluoview 3000 confocal microscope.

\section{Additional files}

Additional file 1: Table S1. Sequences and positions of the 48 oligos
within the Wolbachia 165 and 23 S ribosomal RNA used for Stellaris ${ }^{\circledast}$
rRNA-FISH. (PDF 90 kb)
Additional file 2: Figure S1. Control FISH on Glossina spp. (a) G. m.
morsitans ovary hybridized with Wolbachia (red), and Wigglesworthia
(green) probes in parallel. Wigglesworthia probe does not cross-react with
Wolbachia. (b) Milk gland of Wolbachia-uninfected G. p. palpalis
hybridized with Wigglesworthia (green) and Wolbachia (red) probes. While
Wigglesworthia is recognized in the lumen of the gland, Wolbachia probe
does not give any signal. (c) Triple staining (Wolbachia in red, Wiggle-
sworthia in green, Sodalis in pink) on tetracyline-treated G. m. morsitans
female. None of three probes give a signal in the ovary. Fluorophore
labels of $16-235$ ribosomal RNA probes are FITC (Wigglesworthia), CAL
Fluor Red 590 (Wolbachia), and Quasar 670 (Sodalis). Glossina DNA is
stained in blue (4',6-diamidino-2-phenylindole). (PDF 31389 kb)

Abbreviations

ARM: Wolbachia A-supergroup repeat motif; FISH: fluorescence in situ hybridization; Gbr: G. brevipalpis; Gfu: G. f. fuscipes; Gmc: G. morsitans centralis; Gmm: G. morsitans morsitans; Gpa: G. pallidipes; Gpg: G. palpalis gambiensis; Gpp: G. palpalis palpalis; Gsw: G. swynnertoni

\section{Acknowledgments}

We thank Peter Takač (Slovak Academy of Science) for providing wild type and tetracycline-treated Glossina morsitans morsitans, and Kirsten Senti (University of Veterinary Medicine, Vienna, Austria) for sharing FISH protocols. We also thank Carmen Marin (Insect Pest Control Laboratory, Seibersdorf, Austria) for her great support in tsetse fly handling and maintaining tsetse flies. 


\section{Funding}

This work was supported by two grants (P22634-B17, P28255-B22) from the Austrian Science Fund to W.J.M., and by an Amadeus grant (FR13/2014) from the OeAD/Scientific \& Technological Cooperation to D.I.S. Additional support came from the Coordinated Research Project D42016 'Comparing Rearing Efficiency and Competitiveness of Sterile Male Strains Produced by Genetic, Transgenic or Symbiont-based Technologies' from the International Atomic Energy Agency. The publication cost of this article was funded by the IAEA

\section{Availability of data and materials}

The datasets generated and/or analyzed during the current study are available in the Harvard Dataverse repository, https://doi.org/10.7910/DVN/HMUNOZ.

\section{About this supplement}

This article has been published as part of BMC Microbiology Volume 18 Supplement 1, 2018: Enhancing Vector Refractoriness to Trypanosome Infection. The full contents of the supplement are available online at https://bmcmicrobiol.biomedcentral.com/articles/supplements/volume18-supplement-1.

\section{Authors' contributions}

DIS carried out molecular lab work, performed data analysis, participated in the design of the study and wrote the manuscript; ACP provided flies and helped writing the manuscript; AMM provided flies and helped writing the manuscript; WJM conceived the study, designed the study, and wrote the manuscript. All authors have read and approved the final manuscript.

\section{Ethics approval and consent to participate}

Not applicable.

\section{Consent for publication}

Not applicable.

\section{Competing interests}

The authors declare that they have no competing interests.

\section{Publisher's Note}

Springer Nature remains neutral with regard to jurisdictional claims in published maps and institutional affiliations.

\section{Author details}

1 Department Cell and Developmental Biology, Center for Anatomy and Cell Biology, Medical University of Vienna, Vienna, Austria. ${ }^{2}$ Present Address: Department of Epidemiology of Microbial Diseases, Yale School of Public Health, New Haven, CT, USA. ${ }^{3}$ Insect Pest Control Laboratory, Joint FAO/IAEA Division of Nuclear Techniques in Food and Agriculture, Vienna, Austria.

\section{Published: 23 November 2018}

\section{References}

1. Doudoumis V, Tsiamis G, Wamwiri F, Brelsfoard C, Alam U, Aksoy E, Dalaperas S, Abd Alla A, Ouma J, Takac P, Aksoy S, Bourtzis K. Detection and characterization of Wolbachia infections in laboratory and natural populations of different species of tsetse flies (genus Glossina). BMC Microbiol. 2012. https://doi.org/10.1186/1471-2180-12-S1-S3.

2. Schneider DI, Garschall KI, Parker AG, Abd-Alla AM, Miller WJ. Global Wolbachia prevalence, titer fluctuations and their potential of causing cytoplasmic incompatibilities in tsetse flies and hybrids of Glossina morsitans subgroup species. J Invertebr Pathol. 2013. https://doi.org/10.1016/j.jip.2012.03.024.

3. Schneider DI, Klasson L, Lind AE, Miller WJ. More than fishing in the dark: PCR of a dispersed sequence produces simple but ultrasensitive Wolbachia detection. BMC Microbiol. 2014. https://doi.org/10.1186/1471-2180-14-121.

4. Van Den Abbeele J, Bourtzis K, Weiss B, Cordón-Rosales C, Miller W, Abd-Alla AM, Parker A. Enhancing tsetse fly refractoriness to trypanosome infection--a new IAEA coordinated research project. J Invertebr Pathol 2013; doi:https:// doi.org/10.1016/j.jip.2012.07.020.

5. Miller WJ, Ehrman L, Schneider D. Infectious speciation revisited: impact of symbiont-depletion on female fitness and mating behavior of Drosophila paulistorum. PLoS Pathog. https://doi.org/10.1371/journal.ppat.1001214.

6. Arthofer W, Riegler M, Schneider D, Krammer M, Miller WJ, Stauffer C. Hidden Wolbachia diversity in field populations of the European cherry fruit fly, Rhagoletis cerasi (Diptera, Tephritidae). Mol Ecol. 2009. https://doi.org/10. 1111/j.1365-294X.2009.04321.X.

7. Balmand S, Lohs C, Aksoy S, Heddi A. Tissue distribution and transmission routes for the tsetse fly endosymbionts. J Invertebr Pathol. 2013. https://doi. org/10.1016/j.jip.2012.04.002.

8. Alam U, Medlock J, Brelsfoard C, Roshan P, Lohs C, Balmand S, Carnogursky J, Heddi A, Takac P, Galvani A, Aksoy S. Wolbachia symbiont infections induce strong cytoplasmic incompatibility in the tsetse Fly Glossina morsitans. PLoS Pathog. 2011. https://doi.org/10.1371/journal.ppat.1002415.

9. Doudoumis V, Alam U, Aksoy E, Abd-Alla AM, Tsiamis G, Brelsfoard C, Aksoy S, Bourtzis K. Tsetse-Wolbachia symbiosis: comes of age and has great potential for pest and disease control. J Invertebr Pathol. 2013. https://doi. org/10.1016/j.jip.2012.05.010.

10. Brelsfoard C, Tsiamis G, Falchetto M, Gomulski LM, Telleria E, Alam U, Doudoumis V, Scolari F, Benoit JB, Swain M, Takac P, Malacrida AR, Bourtzis K, Aksoy S. Presence of extensive Wolbachia symbiont insertions discovered in the genome of its host Glossina morsitans morsitans. PLoS Negl Trop Dis. 2014. https://doi.org/10.1371/journal.pntd.0002728.

11. Strunov A, Schneider DI, Albertson R, Miller WJ. Restricted distribution and lateralization of mutualistic Wolbachia in the Drosophila brain. Cell Microbiol. 2017. https://doi.org/10.1111/cmi.12639.

12. Login FH, Balmand S, Vallier A, Vincent-Monégat C, Vigneron A, Weiss-Gayet $M$, Rochat D, Heddi A. Antimicrobial peptides keep insect endosymbionts under control. Science. 2011. https://doi.org/10.1126/science.1209728.

13. Login $\mathrm{FH}$, Heddi $\mathrm{A}$. Insect immune system maintains long-term resident bacteria through a local response. Insect Physiol. 2013. https://doi.org/10. 1016/j.jinsphys.2012.06.015

14. Wang J, Weiss BL, Aksoy S. Tsetse fly microbiota: form and function. Front Cell Infect Microbiol. 2013. https://doi.org/10.3389/fcimb.2013.00069.

15. Benoit JB, Attardo GM, Baumann AA, Michalkova V, Aksoy S. Adenotrophic viviparity in tsetse flies: potential for population control and as an insect model for lactation. Annu Rev Entomol. 2015. https://doi.org/10.1146/ annurev-ento-010814-020834.

16. Arrese EL, Soulages JL. Insect fat body: energy, metabolism, and regulation. Annu Rev Entomol. 2010. https://doi.org/10.1146/annurev-ento-112408-085356.

17. De Vooght L, Caljon G, Van Hees J, Van Den Abbeele J. Paternal transmission of secondary symbiont during mating in the viviparous tsetse fly. Mol Biol Evol. 2015. https://doi.org/10.1093/molbev/msv077.

18. Poinsot D, Montchamp-Moreau C, Merçot H. Wolbachia segregation rate in Drosophila simulans naturally bi-infected cytoplasmic lineages. Heredity. 2000;85(Pt 2):191-8

19. Miller WJ, Riegler M. Evolutionary dynamics of wAu-like Wolbachia variants in Neotropical Drosophila species. Appl Environ Microbiol. 2006. https://doi. org/10.1128/AEM.72.1.826-835.2006.

20. Jeyaprakash A, Hoy MA. Long PCR improves Wolbachia DNA amplification: wsp sequences found in 76\% of sixty-three arthropod species. Insect Mol Biol. 2000;9(4):393-405.
Ready to submit your research? Choose BMC and benefit from:

- fast, convenient online submission

- thorough peer review by experienced researchers in your field

- rapid publication on acceptance

- support for research data, including large and complex data types

- gold Open Access which fosters wider collaboration and increased citations

- maximum visibility for your research: over $100 \mathrm{M}$ website views per year

At $\mathrm{BMC}$, research is always in progress.

Learn more biomedcentral.com/submissions 